\title{
Putative Palytoxin and Its New Analogue, Ovatoxin-a, in Ostreopsis ovata Collected Along the Ligurian Coasts During the 2006 Toxic Outbreak
}

\author{
Patrizia Ciminiello, ${ }^{\text {a }}$ Carmela Dell' Aversano, ${ }^{\text {a }}$ Ernesto Fattorusso, ${ }^{\text {a }}$ \\ Martino Forino, ${ }^{\text {a }}$ Luciana Tartaglione, ${ }^{\text {a }}$ Claudio Grillo, ${ }^{\mathrm{b}}$ and \\ Nunzia Melchiorre ${ }^{\mathrm{b}}$ \\ a Dipartimento di Chimica delle Sostanze Naturali, Università degli Studi di Napoli Federico II, Napoli, Italy \\ b Agenzia Regionale per la Protezione dell'Ambiente Ligure (ARPAL), Dipartimento La Spezia, La Spezia, \\ Italy
}

In this article we report on the liquid chromatography tandem mass spectrometry (LC-MS) investigation of plankton samples collected in the summer of 2006 along the Ligurian coasts, coinciding with a massive bloom of the tropical microalga Ostreopsis ovata. LC-MS analyses indicated the occurrence of putative palytoxin along with a much more abundant palytoxinlike compound never reported so far, which we named ovatoxin-a. On the basis of molecular formula, fragmentation pattern, and chromatographic behavior, the structure of ovatoxin-a appeared to be strictly related to that of palytoxin. We report also on the analysis of cultured $O$. ovata, which was necessary to unequivocally demonstrate that putative palytoxin and ovatoxin-a contained in field samples were actually produced by O. ovata itself. (J Am Soc Mass Spectrom 2008, 19, 111-120) (C) 2008 American Society for Mass Spectrometry

$\mathrm{T}$ The first referenced evidence of the presence of the tropical microalga Ostreopsis spp. in the Mediterranean seawater is dated back to the late 1990s [1]. Since then, these algae have been continuously detected along most of the Mediterranean coastline [2]. They have only occasionally caused cases of human sufferings, until, in summer 2005, a massive proliferation of O. ovata broke out with alarming proportion in Liguria, nearby Genoa, Italy. This phenomenon caught the attention of both national and international media, which delivered breaking news on the event, as hundreds of people required medical attention after exposure to marine aerosol. The symptoms shown by all the patients included fever associated with serious respiratory distress and, in some cases, conjunctivitis. The toxic outbreak reached its climax in coincidence with the highest concentration of O. ovata in seawater and disappeared as the population of this microalga started fading away.

We collected a concentrated plankton sample off Genoa coasts during the toxic outbreak and analyzed it for the most common phycotoxins found in the Mediterranean sea, such as okadaic acid, spirolides, azaspiracids, yessotoxins, paralytic shellfish poisoning toxins, and domoic acid $[3,4]$. We also investigated the occur-

Address reprint requests to Professor E. Fattorusso, Dipartimento di Chimica delle Sostanze Naturali, Università degli Studi di Napoli Federico II, via D. Montesano 49, 80131, Napoli, Italy. E-mail: fattoru@unina.it rence of brevetoxins which, although never reported in the Mediterranean sea, are usually associated with poisonings due to marine aerosols [5]. None of the above toxins was detected in the plankton sample [4].

Some Ostreopsis species produce palytoxin (Figure 1) [6-8], a complex polyhydroxylated compound and one of the most potent marine biotoxins, or palytoxin analogues such as ostreocin-D [9-11] and mascarenotoxins [12]. Structure of ostreocin-D was assigned to 42-hydroxy-3,26-didemethyl-19,44-dideoxypalytoxin, based on NMR data $[9,10]$, and verified by negative-ion fast-atom bombardment collision-induced dissociation tandem mass spectrometry [11]. The charge-remote fragmentations were facilitated by a negative charge introduced to the terminal amino group or to a hydroxyl group at the other terminus of the molecule by reaction of pure ostreocin-D with 2-sulfobenzoic acid cyclic anhydride. Mascarenotoxins [12] were purified and identified as palytoxin-like compounds based on comparison of their mass spectrum profiles and fragmentation patterns with those of a reference standard of palytoxin. Other analogues of palytoxin, namely homo-, bishomo-, neo-, and deoxy-palytoxin were isolated from the soft coral Palythoa tuberculosa [13] but they were not yet proven to be produced by Ostreopsis spp.

To investigate the presence of palytoxin in the plankton sample collected during the Genoa 2005 outbreak, we set up a new method based on liquid chromatography tandem mass spectrometry (LC-MS) for sensitive 


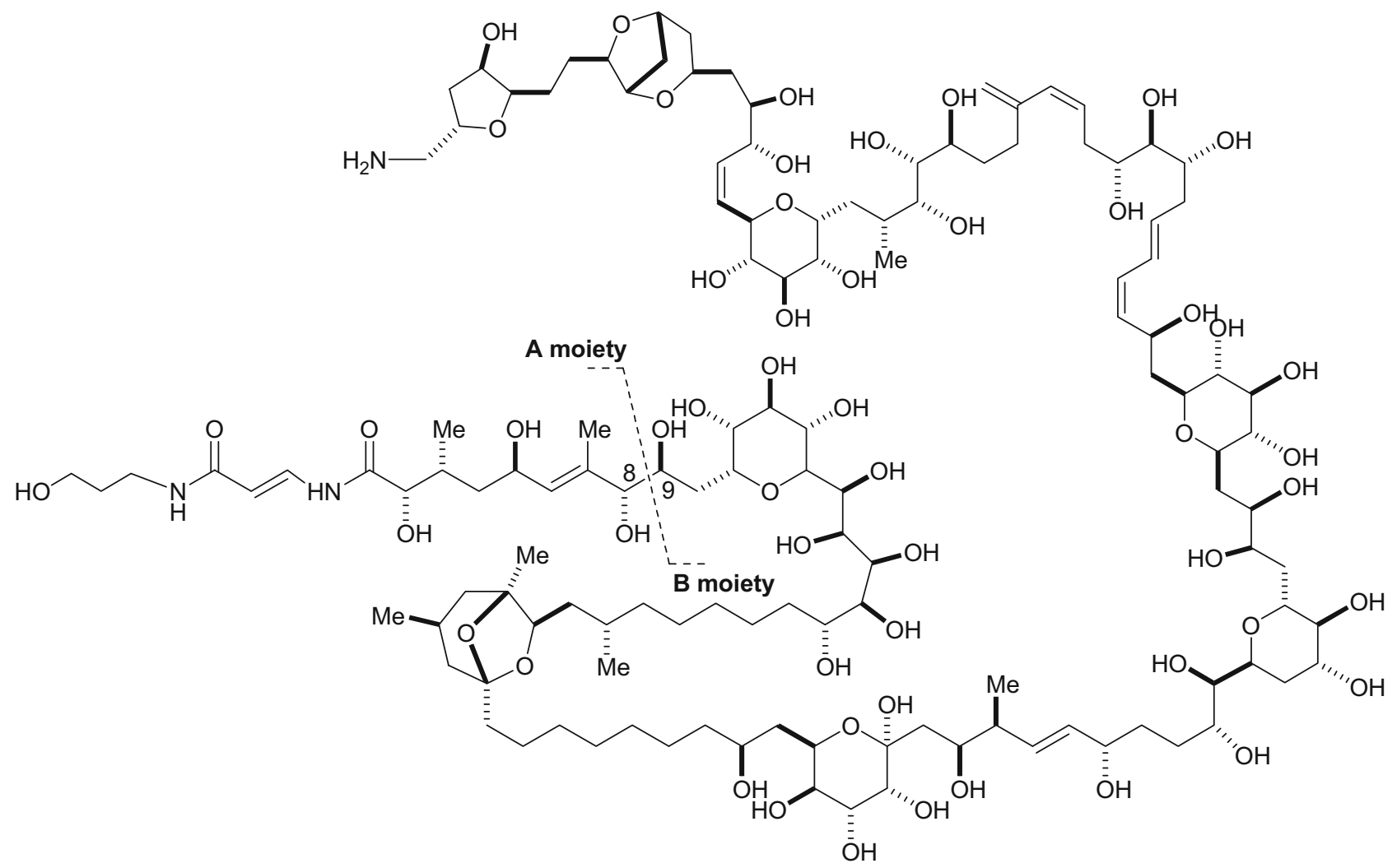

Figure 1. Structure of palytoxin. Cleavage between carbons 8 and 9 originates A and B structural moieties. $\left[\mathrm{M}+\mathrm{H}-\mathrm{B} \text { moiety- } \mathrm{H}_{2} \mathrm{O}\right]^{+}$ion appears at $\mathrm{m} / \mathrm{z} 327.1$ in the full scan $\mathrm{MS}$ spectrum $(\mathrm{DP}=50 \mathrm{~V})$ of palytoxin carried out on the triple quadrupole MS instrument. $\left[\mathrm{M}+\mathrm{H}-\mathrm{A} \text { moiety- } \mathrm{H}_{2} \mathrm{O}\right]^{+}$ion appears at $\mathrm{m} / \mathrm{z} 2318.2749$ in the HR product ion spectrum of the mono-charged ion of palytoxin carried out on the linear ion trap hybrid FTMS instrument.

and selective detection of palytoxin. Relying on the newly developed method, we succeeded in disclosing the involvement of a putative palytoxin in the Genoa 2005 outbreak and assessing its occurrence in the Mediterranean seawater for the very first time [4].

Following this event, in 2006 the presence of O. ovata was monitored along the Ligurian coasts to prevent further human sufferings. Indeed, a remarkable proliferation of O. ovata was detected in the Mediterranean Sea from July through August 2006. Bathing was forbidden in several Italian coastal areas and, thus, the number of people suffering from the toxic outbreak was significantly limited in comparison with the 2005 event [2].

During the 2006 event, we collected field samples of plankton along the Ligurian coasts, where O. ovata bloomed more abundantly. The collected samples were investigated for the presence of palytoxin by the newly developed method and used to create in vitro cultures of the dinoflagellate. In this study, we report on the LC-MS analyses of the plankton samples collected in the summer of 2006, which indicated the presence of putative palytoxin together with a palytoxin-like molecule never reported so far, which we named ovatoxin-a. Furthermore, we report on the analyses of cultured $O$. ovata, which was necessary to unequivocally demonstrate that putative palytoxin and ovatoxin-a contained in field samples were actually produced by $O$. ovata itself.

\section{Experimental}

\section{Reagents}

All organic solvents were of distilled-in-glass grade (Carlo Erba, Milan, Italy). Water was distilled and passed through a MilliQ water purification system (Millipore Ltd., Bedford, MA). Glacial acetic acid (laboratory grade) was purchased from Carlo Erba. Analytical standard of palytoxin was purchased from Wako Chemicals $\mathrm{GmbH}$ (Neuss, Germany) and dissolved in methanol/water (1:1, vol/vol). Matrix-matched standards of palytoxin were prepared as reported previously [4].

\section{Collection and Identification of the Plankton}

The harmful algal bloom occurred in late July through August 2006 along the rocky coasts of Liguria, which present pebbly seabed colonized by macroalgae. Seawater samples were collected by operators of the Agenzia Regionale per la Protezione dell'Ambiente Ligure (ARPAL) and used to determine the qualitative and quan- 
Table 1. Amounts of putative palytoxin and ovatoxin-a ${ }^{a}$ contained in pellets and seawater extracts of $O$. ovata collected along the Ligurian coasts in the summer of 2006

\begin{tabular}{|c|c|c|c|c|c|c|}
\hline \multirow[b]{2}{*}{ Sample } & \multirow[b]{2}{*}{ Collected on } & \multirow{2}{*}{$\begin{array}{l}\text { Cell number } \\
\left(\times 10^{6}\right)\end{array}$} & \multicolumn{2}{|c|}{ Putative palytoxin } & \multicolumn{2}{|c|}{ Ovatoxin-a } \\
\hline & & & $(\mu \mathrm{g})$ & $\mathrm{pg} / \mathrm{cell}$ & $(\mu \mathrm{g})$ & $\mathrm{pg} / \mathrm{cel}$ \\
\hline 1 & July 24 & 41 & 16.5 & 0.40 & 127.6 & 3.11 \\
\hline 2 & July 24 & 24 & 7.0 & 0.29 & 36.4 & 1.51 \\
\hline 3 & August 22 & 22 & 1.9 & 0.09 & 27.8 & 1.26 \\
\hline 4 & August 23 & 14 & 3.1 & 0.22 & 31.6 & 2.26 \\
\hline
\end{tabular}

${ }^{a}$ On account of the structural similarities between palytoxin and ovatoxin-a, we assumed that their molar responses were quite similar. The ovatoxin-a content was determined by direct comparison to a matrix-matched standard of palytoxin [4] at similar concentration injected in the same experimental conditions. Both SIM and MRM experiments were carried out (see Experimental section).

titative composition of phytoplankton. The phytocenoses were characterized after treatment with Lugol solution and sedimentation in Uthermöhl tubes [14]. Analysis on the whole sedimentation chamber with inverted microscope showed that $O$. ovata [15] was the major species present in the sample, together with a few cells of diatoms (Coscinodiscus spp.) and other potentially toxic dinoflagellates, namely Coolia monotis, Prorocentrum lima, and Amphidinium sp. Since O. ovata is a benthonic dinoflagellate, five samples of macroalgae (Rhodophyta, Chlorophyta, and Phaeophyta) covered by a plankton film, were collected and closed underwater in sampling bags (PBI type) in the area of Genoa (samples 1 and 2) and La Spezia (samples 3 and 4). The macroalgal samples were separately transferred to hermetically sealed vessels and the sampling bags were rinsed with filtered (0.22 $\mu \mathrm{m}$ mesh) seawater collected at the sampling sites. The rinsing water was added to the vessels shaking for $5 \mathrm{~min}$ and the cell suspension was filtered through a plankton net (20 $\mu \mathrm{m}$ mesh). The procedure was repeated several times to obtain a plankton pellet that was suspended again in filtered seawater. An aliquot of each sample was treated with Lugol solution and used for cell identification and counting by inverted microscope. The remaining aliquot for each sample was centrifuged using a fixed-angle rotor $\left(r_{\max }\right.$ $9.5 \mathrm{~cm}$ ) at $5000 \mathrm{rpm}$ for $20 \mathrm{~min}$ to separate pellet from seawater, and shipped to the Dipartimento di Chimica delle Sostanze Naturali, Università degli Studi di Napoli Federico II, for chemical analyses.

\section{Cultures of O. ovata}

Cultures of $O$. ovata were obtained from sample 1 collected at the site most affected by the phenomenon of algal bloom on July 24, 2006. Single cells were isolated by micromanipulation at the inverted microscope and transferred in sterile slabs equipped with traps $(1.5 \mathrm{~cm}$ diameter). They were grown in conditions close to the natural ones, as reported in literature for Ostreopsidaceae cultures (temperature $25^{\circ} \mathrm{C}$, luminous existence $2000 \mathrm{l} \times, 16: 8 \mathrm{~h}$ light-to-dark cycle) [16]. The standard K-Keller medium was used, which supplies $\mathrm{NaNO}_{3}$ and $\mathrm{NH}_{4} \mathrm{Cl}$ as nitrogen sources, and $\beta$-glycerophosphate in place of $\mathrm{NaH}_{2} \mathrm{PO}_{4}$. Gradually, cultures were transferred to larger flasks, up to
$500 \mathrm{~mL}$. The algal pellets $\left(16 \times 10^{6}\right.$ cell $)$ were collected during late exponential growth phases, on 14th day, by a disposable vacuum filtration system (Stericup; Millipore) on $0.22 \mu \mathrm{m}$ cellulose filters. Both pellets and growth medium were stored at $-20{ }^{\circ} \mathrm{C}$ until shipment.

\section{Extraction}

Each field (or cultured) pellet sample was sonicated with $8 \mathrm{~mL}$ of a methanol/water (1:1, vol/vol) solution for $3 \mathrm{~min}$ in pulse mode, while cooling in ice bath. The mixture was centrifuged at $5500 \mathrm{rpm}$ for $30 \mathrm{~min}$, the supernatant was decanted, and the pellet was washed twice with $8 \mathrm{~mL}$ of methanol/water $(1: 1, \mathrm{vol} / \mathrm{vol})$. The extracts were combined and the volume was adjusted to $30 \mathrm{~mL}$ with extracting solvent. The obtained mixture was analyzed directly by LC-MS ( $5 \mu \mathrm{L}$ injected). Each seawater (or growth medium) sample was extracted twice with an equal volume of butanol. The butanol layer was evaporated to dryness, dissolved in $5 \mathrm{~mL}$ of methanol/water (1:1, vol/vol), and analyzed directly by LC-MS (5 $\mu \mathrm{L}$ injected).

\section{Liquid Chromatography-Mass Spectrometry}

LC-MS analyses were accomplished by using a $3 \mu \mathrm{m}$ Gemini C18 $(150 \times 2.00 \mathrm{~mm})$ column (Phenomenex, Torrance, CA) maintained at room temperature and eluted at $0.2 \mathrm{~mL} \mathrm{~min}^{-1}$ with water (Eluent A) and 95\% acetonitrile/water (Eluent B), both containing $30 \mathrm{mM}$ acetic acid. The following gradient systems were used: a fast gradient elution (20\% to $100 \%$ B over $10 \mathrm{~min}$ and hold $5 \mathrm{~min}$ ), which allowed elution of palytoxin at 6.60 min, and a slow gradient elution $(20 \%$ to $50 \%$ B over 20 $\mathrm{min}, 50 \%$ to $80 \%$ B over $10 \mathrm{~min}, 80 \%$ to $100 \%$ B in $1 \mathrm{~min}$, and hold $5 \mathrm{~min}$ ), which allowed chromatographic separation between palytoxin ( $\mathrm{Rt}=11.08 \mathrm{~min}$ ) and ovatoxin-a $(\mathrm{Rt}=11.48 \mathrm{~min})$. Mass spectral experiments at unit resolution $(\mathrm{FWHM}=0.7 \pm 0.1 \mathrm{Th}$ ) were carried out on the following LC-MS systems: (1) an Agilent 1100 LC binary system (Palo Alto, CA) coupled to a PE-SCIEX API 2000 triple-quadrupole MS equipped with a Turbospray source (Concorde, ON, Canada); (2) a Surveyor MS plus LC quaternary system coupled to an LCQ DECA XP ion trap MS equipped with an OPTON ESI 
a)

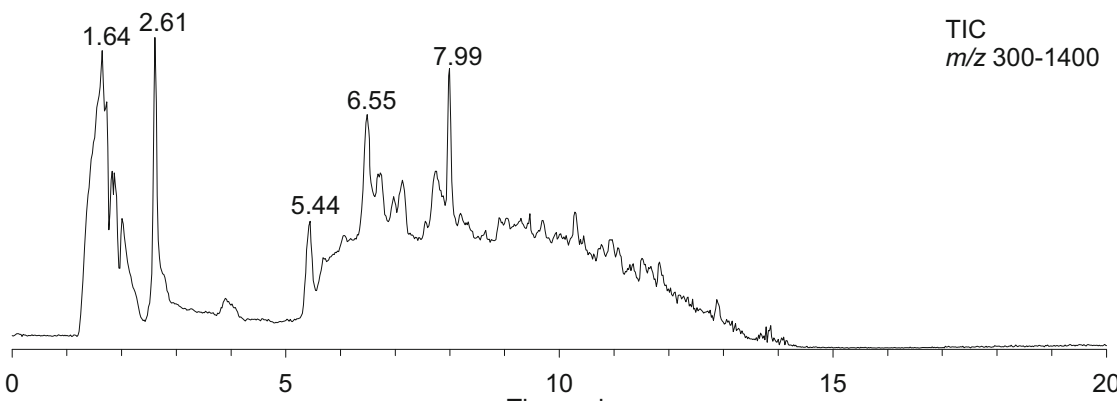

c)

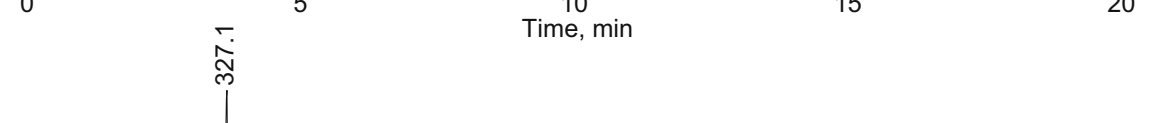

c) b)

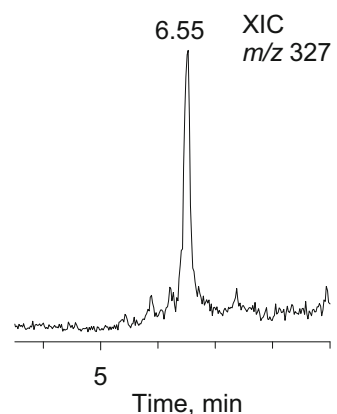

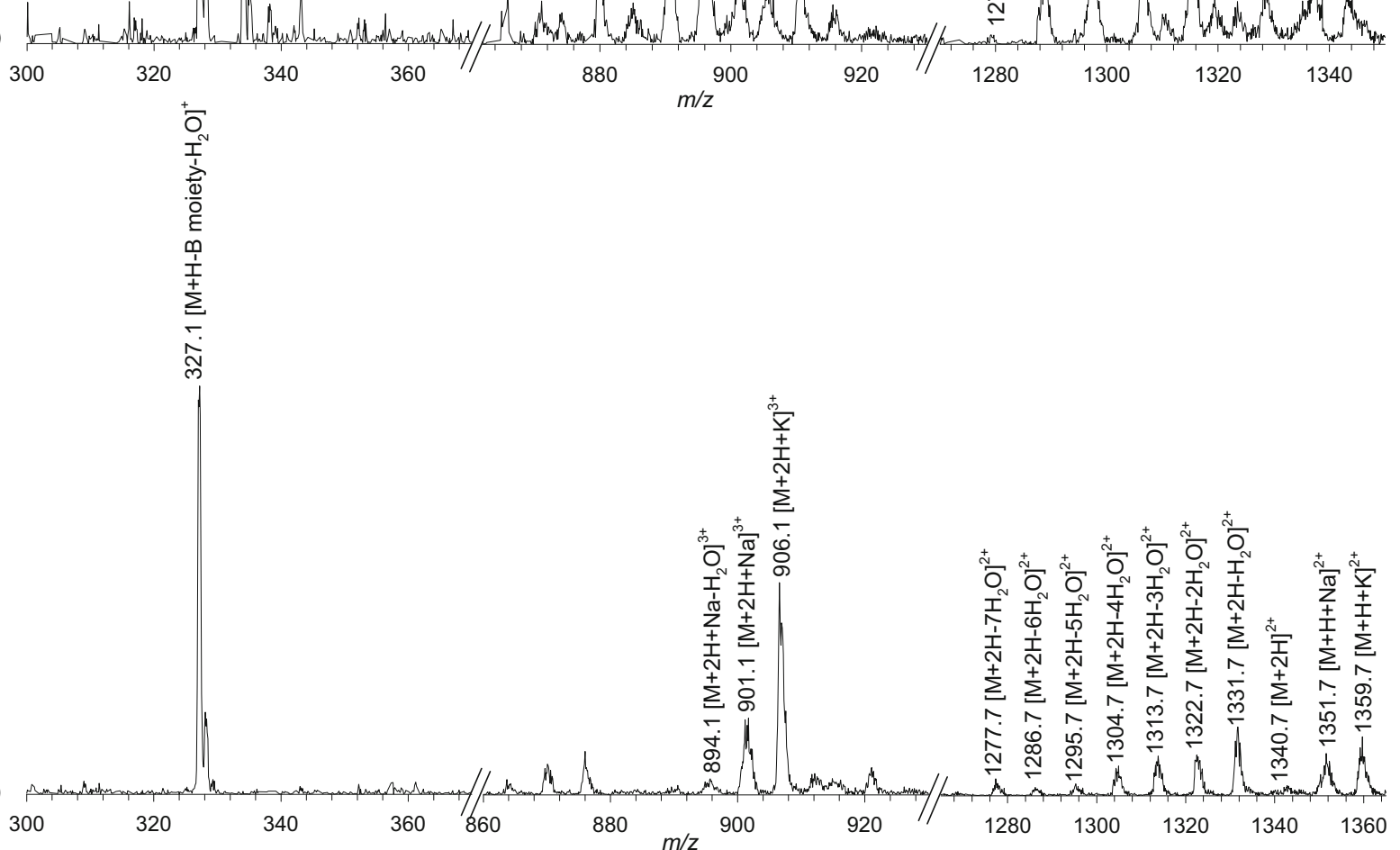

Figure 2. (a) Total ion chromatogram (TIC) of sample 1; (b) related extracted ion chromatogram (XIC) at $\mathrm{m} / \mathrm{z} 327.1$; (c) full scan MS spectrum associated with the peak eluting at $6.55 \mathrm{~min}$; (d) full scan MS spectrum of palytoxin standard. LC-MS analyses of sample 1 and palytoxin standard were acquired on the triple quadrupole MS instrument in positive full scan MS mode (DP $=50 \mathrm{~V}$ ), by using the following LC conditions: a $3 \mu \mathrm{m}$ Gemini C18 $(150 \times 2.00 \mathrm{~mm})$ column eluted at $0.2 \mathrm{~mL} \mathrm{~min}^{-1}$ with $\mathrm{A}=$ water and $\mathrm{B}=95 \%$ acetonitrile/water, both eluents containing $30 \mathrm{mM}$ acetic acid. A fast gradient elution ( $20 \%$ to $100 \%$ B over $10 \mathrm{~min}$ and hold $5 \mathrm{~min}$ ) was used. Assignment of bi-charged and tri-charged ion clusters was based on high-resolution MS data, since the low-resolution settings on the mass spectrometer and annotation of the centroids of unresolved multiply charged ions could lead to misleading compositions [4]. 
a)
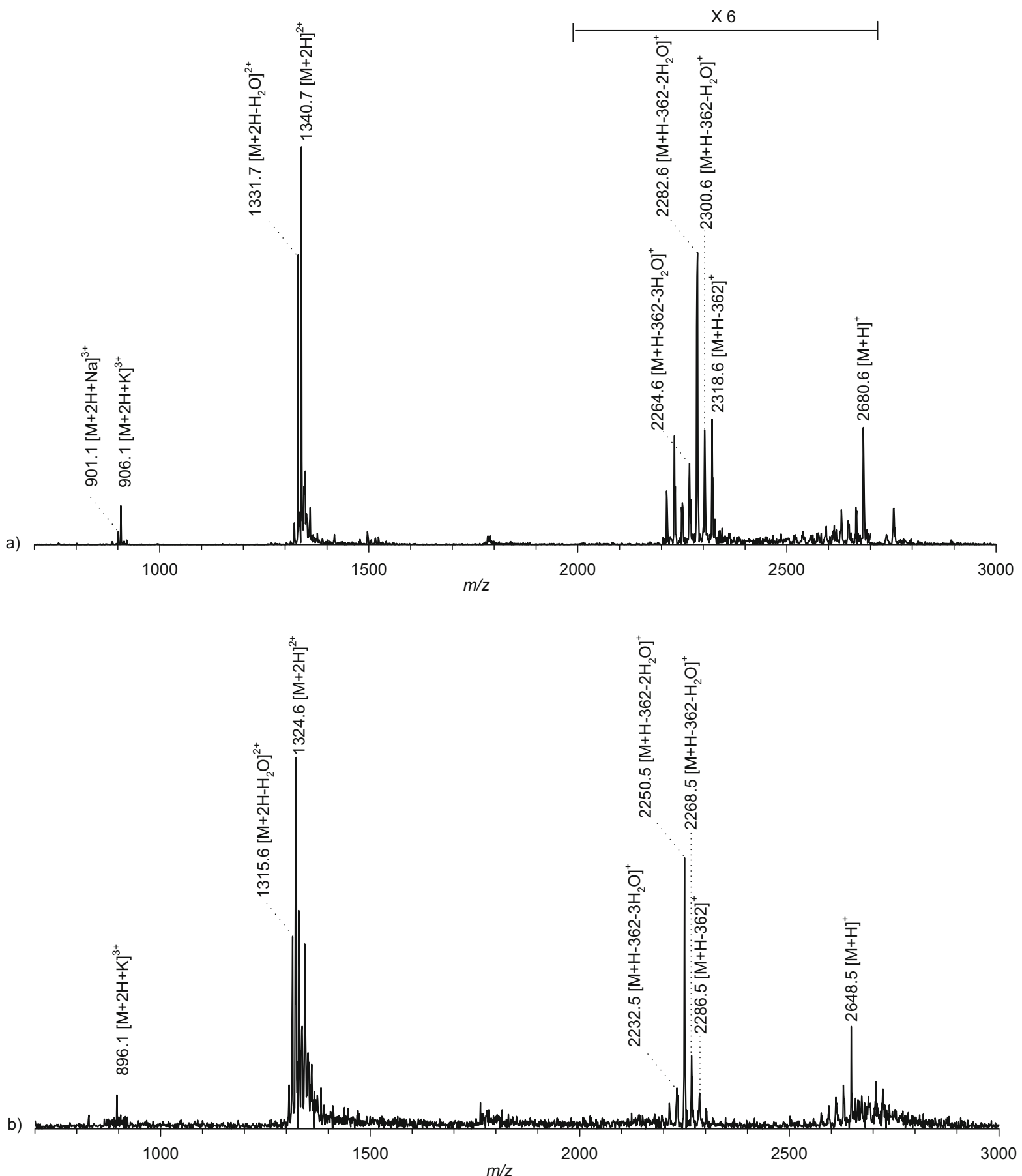

Figure 3. (a) Full scan MS spectrum of palytoxin standard; (b) full scan MS spectrum of the peak eluting at $6.55 \mathrm{~min}$ in the LC-MS analysis of sample 1. The spectra were acquired on the ion trap MS instrument in positive ion mode by using the same LC conditions as in Figure 2. Assignment of bi-charged and tri-charged ion clusters was based on high-resolution MS data, since the lowresolution settings on the mass spectrometer and annotation of the centroids of unresolved multiply charged ions could lead to misleading compositions [4].

source (Thermo-Fisher, San Jose, CA). High-resolution (HR) MS and product ion spectra were acquired by using a Surveyor MS plus LC quaternary system coupled to a linear ion trap LTQ Orbitrap XL hybrid Fourier Transform MS (FTMS) equipped with an ESI ION MAX source (Thermo-Fisher, San Jose, CA).

\section{Triple Quadrupole MS Experiments}

Full scan MS experiments in the range $m / z$ 300-1400 were carried out in positive ion mode by using the following source settings: a turbogas temperature of $300{ }^{\circ} \mathrm{C}$, an ionspray voltage of $5500 \mathrm{~V}$, a declustering 


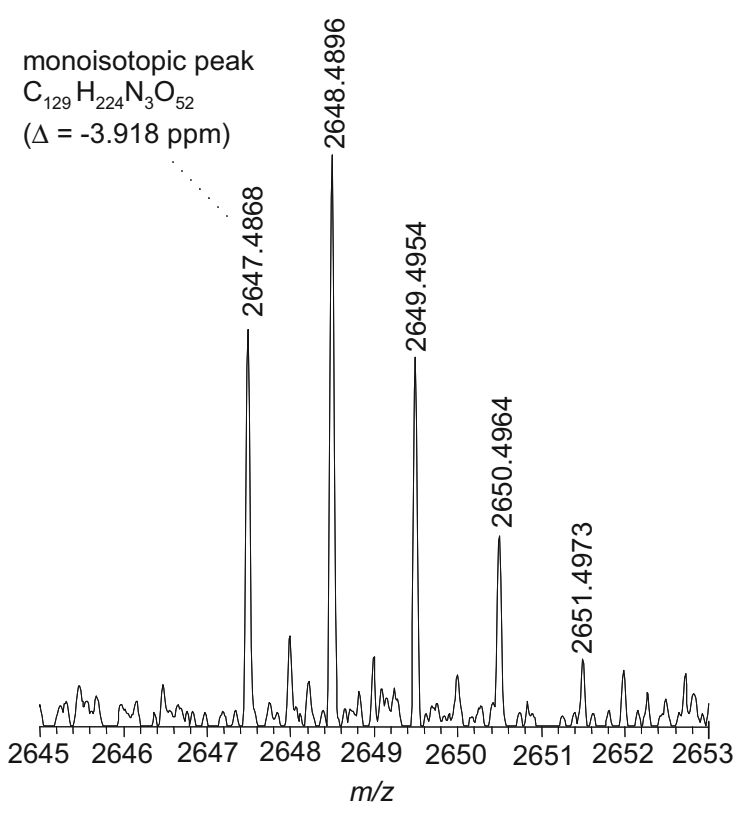

Figure 4. Mass scale expansion of the $m / z 2645-2653$ region of the HRMS spectrum of ovatoxin-a acquired on the linear ion trap hybrid FTMS instrument in positive ion mode by using the same LC conditions as in Figure 2.

potential (DP) of $8 \mathrm{~V}(50 \mathrm{~V}$ in the in-source fragmentation experiments), a focusing potential (FP) of $350 \mathrm{~V}$, and an entrance potential (EP) of $11 \mathrm{~V}$. Multiple reaction monitoring (MRM) experiments were carried out by using a collision energy of $50 \mathrm{eV}$, a cell exit potential (CXP) of 10 $\mathrm{V}$, and monitoring the transitions $\mathrm{m} / \mathrm{z} 1340.7 \rightarrow 327.1$ $(\mathrm{DP}=8 \mathrm{~V}), 1331.7 \rightarrow 327.1(\mathrm{DP}=8 \mathrm{~V})$, and $906.1 \rightarrow$ $327.1(\mathrm{DP}=50 \mathrm{~V})$ for palytoxin and the transitions $\mathrm{m} / \mathrm{z}$ $1324.7 \rightarrow 327.1(\mathrm{DP}=8 \mathrm{~V}), 1315.7 \rightarrow 327.1(\mathrm{DP}=8 \mathrm{~V})$, $896.1 \rightarrow 327.1(\mathrm{DP}=50 \mathrm{~V})$ for ovatoxin-a. The dwell time was set to achieve a total scan time of $1 \mathrm{~s}$. The transitions $\mathrm{m} / \mathrm{z} 1340.7 \rightarrow 327.1$ and $1324.7 \rightarrow 327.1$ were used for quantitation of putative palytoxin and ovatoxin-a, respectively. Quantitative results were obtained by direct comparison of peak areas to matrix-matched standard solutions of palytoxin at similar concentration injected in the same experimental conditions [4]. MRM quantitative results obtained for ovatoxin-a were confirmed by selected ion monitoring (SIM) experiments. The following ions were monitored for ovatoxin-a $\mathrm{m} / \mathrm{z}$ 890.1, 896.1, 1297.7, 1306.7, 1315.7, 1324.7, 1335.7, 1343.7 whereas the following ions were monitored for palytoxin $\mathrm{m} / \mathrm{z}$ 906.1, 901.1, 1313.7, 1322.7, 1331.7, 1340.7, 1351.7, 1359.7. The obtained peak areas for ovatoxin-a were summed and compared with ions sum of standard solutions of palytoxin injected in the same experimental conditions.

\section{Ion Trap MS Experiments}

Full scan MS experiments in the range $m / z \quad 700-3000$ were carried out in positive ion mode by using a spray voltage of $7.5 \mathrm{kV}$, a capillary temperature of $200^{\circ} \mathrm{C}$, a capillary voltage of $39 \mathrm{~V}$, a sheath gas and an auxiliary gas flow of 50 and 10 (arbitrary units), respectively.

\section{Linear Ion Trap Hybrid FTMS Experiments}

HRMS experiments (positive ions) were acquired in the range $\mathrm{m} / \mathrm{z} 600-3000$ at the 100,000 resolving power setting by using the following source settings: a spray voltage of $3.8 \mathrm{kV}$, a capillary temperature of $260^{\circ} \mathrm{C}$, a capillary voltage of $49 \mathrm{~V}$, a sheath gas and an auxiliary gas flow of 50 and 10 (arbitrary units), respectively. High-resolution product ion spectra were acquired at the 60,000 resolving power setting by fragmenting the $[\mathrm{M}+\mathrm{H}]^{+}$ion of both palytoxin $(\mathrm{m} / \mathrm{z} 2680.5)$ and ovatoxin-a $(\mathrm{m} / \mathrm{z} 2648.5)$ with a collision energy of $25 \%$, an activation $Q$ of 0.250 , and an activation time of $30 \mathrm{~ms}$. Calculation of elemental formula of ovatoxin-a and its fragments contained in the product ion spectrum was performed by using the mono-isotopic ion peak of each ion cluster.

\section{Results and Discussion}

Four plankton samples were collected in summer 2006 along the Ligurian coasts in the area of Genoa (1 and 2) and La Spezia (3 and 4). Analyses with inverted microscope showed that $O$. ovata was the dominant species in the collected samples [15], while just a few cells of diatoms (Coscinodiscus spp.) and other potentially toxic dinoflagellates, namely Coolia monotis, Prorocentrum lima, and Amphidinium sp., were present in seawater.

The collected plankton samples were independently centrifuged to separate pellets from seawater, which were extracted as reported in the Experimental section. The crude extracts were directly analyzed by the newly developed method for detection of palytoxin [4] using an ESI-triple quadrupole MS instrument operating in the mass range $0-1800$. Chromatographic separation was accomplished on a reversed-phase column by using a mobile phase containing water/acetonitrile, 30 $\mathrm{mM}$ acetic acid and a fast gradient elution, which allowed the early elution of palytoxin standard at 6.60 min. Multiple reaction monitoring (MRM) experiments were carried out by selecting the transition (precursor ion $\rightarrow$ product ion) reported for palytoxin in our previous paper [4] $(\mathrm{m} / \mathrm{z} 1340.7 \rightarrow 327.1)$ together with two additional transitions at $\mathrm{m} / \mathrm{z} 1331.7 \rightarrow 327.1$ and $906.1 \rightarrow 327.1$, for further confirmation. The product ion at $\mathrm{m} / \mathrm{z} 327.1$ arises from the cleavage between the carbons 8 and 9 of palytoxin [8] and the additional loss of a water molecule; it can be assigned to the $[\mathrm{M}+\mathrm{H}-$ B moiety- $\left.\mathrm{H}_{2} \mathrm{O}\right]^{+}$ion (Figure 1). This ion dominates the product ion spectrum of palytoxin, whatever the precursor ion used, either the $[\mathrm{M}+2 \mathrm{H}]^{2+}$ ion at $m / z$ 1340.7, the $\left[\mathrm{M}+2 \mathrm{H}-\mathrm{H}_{2} \mathrm{O}\right]^{2+}$ ion at $\mathrm{m} / \mathrm{z} 1331.7$, or the $[\mathrm{M}+2 \mathrm{H}+\mathrm{K}]^{3+}$ ion at $\mathrm{m} / \mathrm{z}$ 906.1.

Results of MRM analyses of the plankton collected in 2006 paralleled those obtained on the 2005 plankton samples. The presence of putative palytoxin was shown 


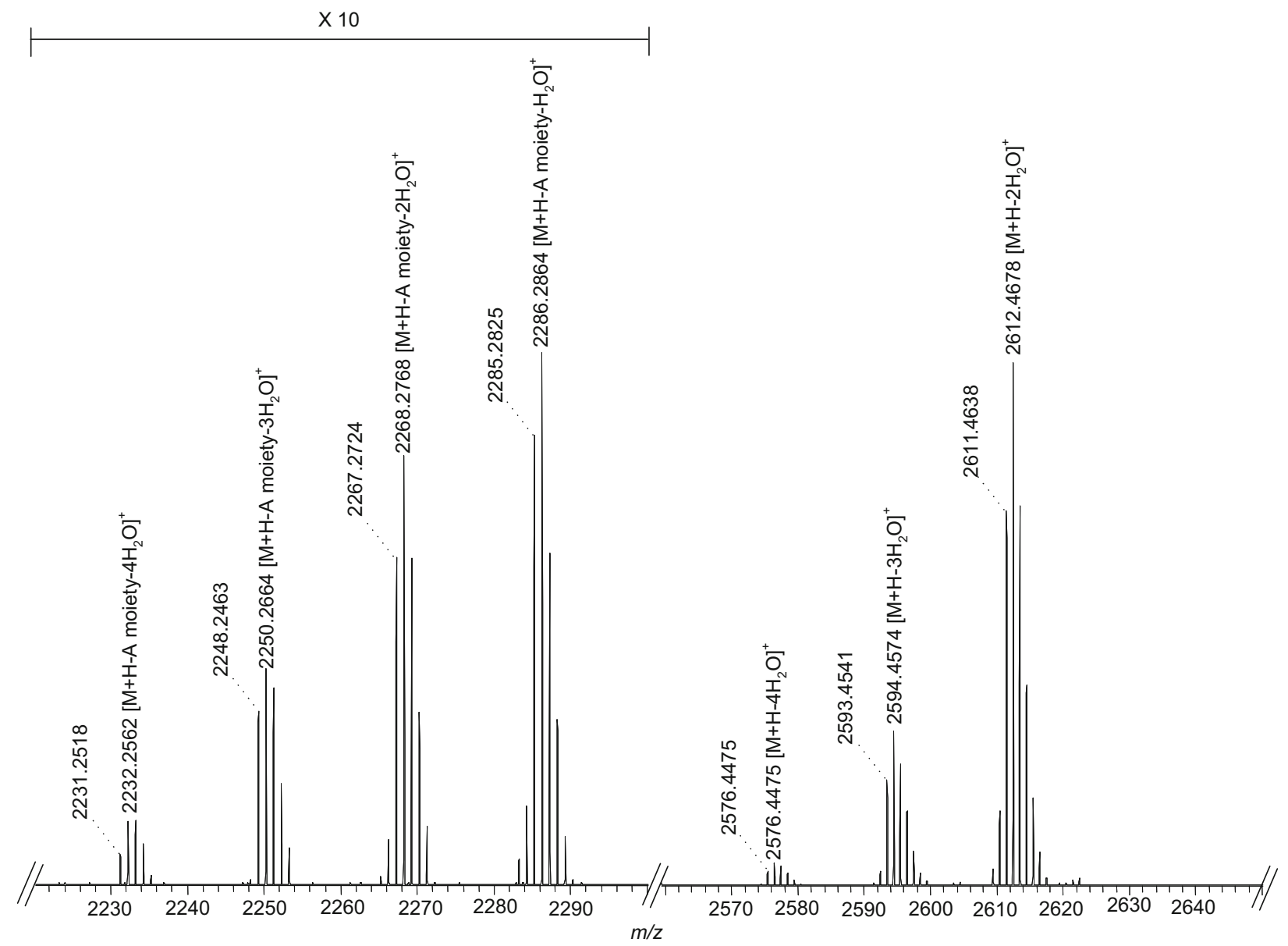

Figure 5. Mass scale expansions of the $m / z 2220-2300$ and $m / z 2560-2650$ regions of the HR product ion spectrum of ovatoxin-a acquired on the linear ion trap hybrid FTMS instrument by using the $[\mathrm{M}+\mathrm{H}]^{+}$ion at $m / z 2648.5$ as precursor and the same LC conditions as in Figure 2. Dotted lines indicate the mono-isotopic ion peaks.

in all the samples, $1-4$, by peaks eluting at $6.55 \mathrm{~min}$, which closely matched those of a reference sample of palytoxin in retention time, fragmentation, and ion ratios. The amounts of putative palytoxin contained in samples 1-4 are reported in Table 1 . The toxin content was determined by direct comparison with matrixmatched standards of palytoxin at similar concentration. The use of matrix-matched standards is required for accurate quantitation since palytoxin ionization suffers from some matrix suppression effect, which is analyte concentration-dependent [4].

Basing on MRM quantitative results, sample 1 appeared to contain a relatively high amount of putative palytoxin, which allowed a more detailed and comprehensive MS investigation. To look for additional palytoxin analogues in the sample, we carried out a full scan MS experiment on the triple quadrupole MS in the range 300-1400 $\mathrm{u}$ by favouring the in-source formation of the fragment ion $\left[\mathrm{M}+\mathrm{HB} \text { moiety- } \mathrm{H}_{2} \mathrm{O}\right]^{+}$at $m / z$ 327.1. This ion is generated in the fragmentation experiments of palytoxin itself as well as in those of some other palytoxin-like compounds [12, 13]. Preliminary experiments showed that in-source formation of ion at $\mathrm{m} / \mathrm{z}$
327.1 depends basically on front end potentials used, mostly declustering potential (DP), whereas it is not affected by variation in turbo ion spray temperature. Thus a DP of $50 \mathrm{~V}$ and a spray temperature of $300{ }^{\circ} \mathrm{C}$ were used.

The total ion chromatogram (TIC) of sample 1 (Figure 2a) appeared very complex, which was not surprising since the analyzed sample was a crude extract, not subjected to any clean-up. Thus, we extracted the ion at $\mathrm{m} / \mathrm{z} 327.1$ by obtaining an extracted ion chromatogram (XIC) (Figure 2b) dominated by a peak at an elution time $(6.55 \mathrm{~min})$ close to that of the palytoxin standard (6.60 $\mathrm{min}$ ). The associated mass spectrum (Figure 2c) contained tri-charged and bi-charged ion clusters in the range 850-930 and 1270-1370, respectively, together with the base peak at $m / z$ 327.1. An accurate comparison between this MS spectrum and that of the palytoxin standard (Figure 2d) indicated that, although both spectra contained the ion peak at $\mathrm{m} / \mathrm{z} 327.1$, they presented similar tri-charged and bi-charged ion clusters, which basically differed only for $\mathrm{m} / \mathrm{z}$ absolute values. This evidence pointed to the presence in sample 1 of a 


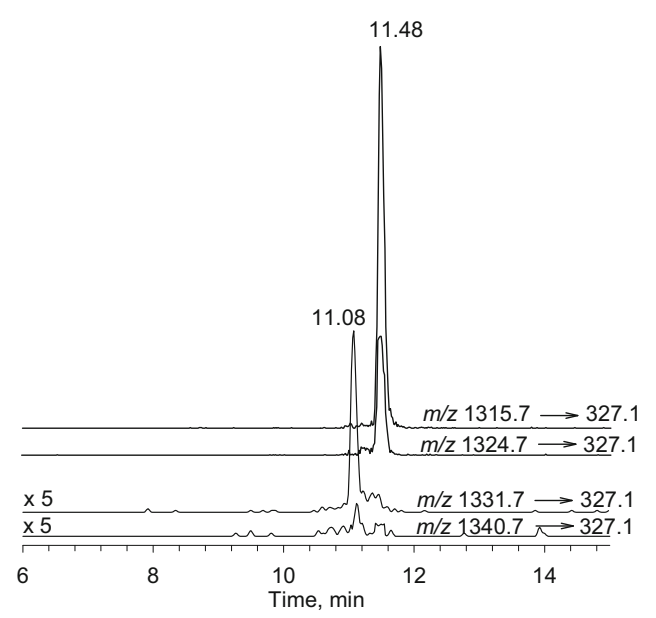

Figure 6. LC-MS analysis of sample 1 acquired on the triple quadrupole MS instrument in positive MRM mode. The MRM transitions $\mathrm{m} / \mathrm{z} 1340.7 \rightarrow 327.1$ and $1331.7 \rightarrow 327.1$ were used to detect palytoxin. The MRM transitions $\mathrm{m} / \mathrm{z} 1324.7 \rightarrow 327.1$ and $1315.1 \rightarrow 327.1$ were selected for detection of ovatoxin-a. The following LC conditions were used: a $3 \mu \mathrm{m}$ Gemini C18 $(150 \times$ $2.00 \mathrm{~mm}$ ) column eluted at $0.2 \mathrm{~mL} \mathrm{m^{-1 }}$ with $\mathrm{A}=$ water and $\mathrm{B}=$ $95 \%$ acetonitrile/water, both eluents containing $30 \mathrm{mM}$ acetic acid. A slow gradient elution (20\% to $50 \%$ B over $20 \mathrm{~min}, 50 \%$ to $80 \%$ B over $10 \mathrm{~min}, 80 \%$ to $100 \% \mathrm{~B}$ in $1 \mathrm{~min}$, and hold $5 \mathrm{~min}$ ) was used. Under the used LC conditions, putative palytoxin eluted at 11.08 $\mathrm{min}$, while ovatoxin-a eluted at $11.48 \mathrm{~min}$.

palytoxin-like molecule that didn't correspond to palytoxin itself. We named it ovatoxin-a.

Additional information was obtained by full scan MS experiments run on an ESI-ion trap-MS instrument operating in the mass range 50-4000 u. Such experiments were carried out on both sample 1 extract and palytoxin standard. Under the used ionization conditions, the MS spectrum of palytoxin (Figure 3a) was dominated by the $[\mathrm{M}+2 \mathrm{H}]^{2+}$ ion peak at $\mathrm{m} / z$ 1340.7, while only one water molecule was lost from the $[\mathrm{M}+2 \mathrm{H}]^{2+}$, and the fragment ion at $\mathrm{m} / \mathrm{z} 327.1$ was completely lacking. Tri-charged ions $[\mathrm{M}+2 \mathrm{H}+\mathrm{Na}]^{3+}$ and $[\mathrm{M}+2 \mathrm{H}+\mathrm{K}]^{3+}$ were present at $\mathrm{m} / \mathrm{z} 901.1$ and 906.1, respectively. In the upper region of the MS spectrum, the mono-charged $[\mathrm{M}+\mathrm{H}]^{+}$ion of palytoxin was present at $\mathrm{m} / \mathrm{z} 2680.6$ and an additional ion peak was present at $\mathrm{m} / \mathrm{z} 2318.6$, followed by ion peaks attributable to subsequent losses of three water molecules. The ion at $m / z 2318.6$ was likely due to an in-source fragmentation of the $[\mathrm{M}+\mathrm{H}]^{+}$ion of palytoxin by loss of $362 \mathrm{u}$. The different degree of front end fragmentation of palytoxin observed on turbospray triple quadrupole MS and ESI ion trap MS could be likely due to different geometry of ionization sources and ionization parameters used.

The TIC of sample 1 showed a chromatographic peak at a retention time close to that of palytoxin. The associated MS spectrum (Figure 3b), interpreted in the light of that of palytoxin (Figure 3a), presented a mono-charged ion at $m / z 2648.5$, which was assigned to the $[\mathrm{M}+\mathrm{H}]^{+}$ion, a dominant bi-charged ion peak at $\mathrm{m} / \mathrm{z}$
1324.6, $[\mathrm{M}+2 \mathrm{H}]^{2+}, \mathrm{a}\left[\mathrm{M}+2 \mathrm{H}-\mathrm{H}_{2} \mathrm{O}\right]^{2+}$ ion at $m / z$ 1315.6, and a tri-charged ion at $m / z$ 896.1, $[\mathrm{M}+2 \mathrm{H}+\mathrm{K}]^{3+}$. Similarly to palytoxin, the fragment ion at $\mathrm{m} / \mathrm{z} 327.1$ was lacking in the spectrum but an additional ion peak was present at $\mathrm{m} / \mathrm{z} 2286.5$, corresponding to a $362 \mathrm{u}$ neutral loss from the $[\mathrm{M}+\mathrm{H}]^{+}$ion, together with ion peaks due to subsequent losses of three water molecules. These data, particularly the $\mathrm{m} / \mathrm{z}$ values of tri-charged and bi-charged ions, which corresponded to ion peaks observed in MS spectrum obtained on the triple quadrupole MS system (Figure 2c), indicated that the compound was actually ovatoxin-a.

The next step was to establish the exact mass of ovatoxin-a and its fragments to assign the molecular formula and to gain additional information on the structure of ovatoxin-a. Thus, LC-MS and product ion MS experiments were run for both palytoxin standard and sample 1 on a linear ion trap hybrid FTMS instrument in the mass range $600-3000 \mathrm{u}$ at a resolving power of 100,000. The mono-charged ion cluster in the HRMS spectrum of ovatoxin-a presented a mono-isotopic ion peak $[\mathrm{M}+\mathrm{H}]^{+}$at $\mathrm{m} / \mathrm{z} 2647.4868$ (Figure 4), which allowed to infer the molecular formula $\mathrm{C}_{129} \mathrm{H}_{223} \mathrm{~N}_{3} \mathrm{O}_{52}(\Delta$ $=-3.918 \mathrm{ppm}$ ) to the molecule. Further confirmation for such elemental composition was provided by the exact masses of bi-charged (mono-isotopic ion peak at $\mathrm{m} / \mathrm{z} 1335.2429$ for $\mathrm{C}_{129} \mathrm{H}_{224} \mathrm{~N}_{3} \mathrm{NaO}_{52,} \Delta=-0.174 \mathrm{ppm}$ ) and tri-charged (mono-isotopic ion peak at $\mathrm{m} / \mathrm{z} 890.4937$ for $\mathrm{C}_{129} \mathrm{H}_{225} \mathrm{~N}_{3} \mathrm{NaO}_{52,}, \Delta=-4.657$ ppm) ions. So, ovatoxin-a presents two oxygen atoms less than palytoxin $\left(\mathrm{C}_{129} \mathrm{H}_{223} \mathrm{~N}_{3} \mathrm{O}_{54}\right)$.

We also recorded an LC-HR product ion spectrum on sample 1 by selecting the mono-charged ion of ovatoxin-a as precursor ion. The obtained spectrum (Figure 5) contained abundant peaks at $\mathrm{m} / \mathrm{z} 2612.4678$, 2594.4574, and 2576.4475, which corresponded to subsequent losses of two, three, and four water molecules from the $[\mathrm{M}+\mathrm{H}]^{+}$ion, respectively. In addition, a structurally interesting fragment ion was present at $\mathrm{m} / \mathrm{z}$ 2286.2864 (mono-isotopic ion at $\mathrm{m} / \mathrm{z} 2285.2825$ ), which was followed by three ion peaks attributable to subsequent losses of water molecules. The ion at $\mathrm{m} / \mathrm{z}$ 2286.2864 was due to the loss of $362.2043 \mathrm{u}$ from the precursor ion, corresponding to a $\mathrm{C}_{16} \mathrm{H}_{30} \mathrm{~N}_{2} \mathrm{O}_{7}(\Delta=$ $-2.765 \mathrm{ppm}$ ) neutral fragment.

In the same experimental conditions, we recorded an LC-HR product ion spectrum of palytoxin standard by using the mono-charged ion of palytoxin as precursor ion. The obtained spectrum paralleled that of ovatoxin-a in the presence of ions ( $\mathrm{m} / \mathrm{z} 2644.4552,2626.4452$, and 2608.4343) due to loss of two, three, and four water molecules, respectively, and an ion $(m / z 2318.2749)$ due to neutral loss of $362.2036 \mathrm{u}\left(\mathrm{C}_{16} \mathrm{H}_{30} \mathrm{~N}_{2} \mathrm{O}_{7}\right)$ accompanied by three losses of water molecules. The neutral fragment $\mathrm{C}_{16} \mathrm{H}_{30} \mathrm{~N}_{2} \mathrm{O}_{7}$ originates from cleavage between carbons 8 and 9 and corresponds to A moiety of palytoxin with an additional molecule of water (Figure 1).

Comparison of MS data of ovatoxin-a and palytoxin suggests that the two molecules share the same part 
structure A as indicated by (1) the common loss of a $\mathrm{C}_{16} \mathrm{H}_{30} \mathrm{~N}_{2} \mathrm{O}_{7}$ part structure from the mono-charged ions emerging from the experiments performed on ion trap MS and linear ion trap hybrid FTMS instruments; (2) fragment ion at $m / z 327.1$ contained in MS spectra of both compounds run on the triple quadrupole MS instrument. Thus, structural differences between ovatoxin-a and palytoxin likely lie in the part structure B (Figure 1).

To gain information about the relative abundance of ovatoxin-a and putative palytoxin in the plankton samples, we set up an LC-MS experiment by using a slow gradient elution, which allowed to chromatographically separate the two compounds (see the Experimental section). MS detection was accomplished in MRM mode on the triple quadrupole MS instrument by monitoring the transitions $\mathrm{m} / \mathrm{z} 1340.7 \rightarrow 327.1,1331.7 \rightarrow 327.1$ for putative palytoxin and $m / z 1324.7 \rightarrow 327.1,1315.7 \rightarrow$ 327.1 for ovatoxin-a. The best results are shown in Figure 6. Under the chromatographic conditions used, putative palytoxin eluted at the same retention time as that of the palytoxin standard, namely $11.08 \mathrm{~min}$, while ovatoxin-a eluted at $11.48 \mathrm{~min}$. On account of the evident structural similarities between the two compounds, we assumed that their molar responses were quite similar. However, since differences in adduct formation could greatly affect relative intensities of different adduct ions, MRM quantitative results obtained for ovatoxin-a were corroborated by SIM experiments. All significant ions contained in full scan MS spectra of ovatoxin-a were monitored, the obtained peak areas for ovatoxin-a were summed, and compared with ion sum of corresponding ions monitored for palytoxin. SIM results were in full agreement with MRM data. The calculated amount of ovatoxin-a in samples 1-4 are reported in Table 1.

Comparison between putative palytoxin and ovatoxin-a contents suggested that this latter was by far the predominant palytoxin-like compound in the 2006 plankton.

Cultures of $O$. ovata were obtained from shares of microalgal pellets coming from the washing of macroalgae collected in correspondence of the site interested by the phenomenon of algal bloom in July 2006. Cells were cultured at $25^{\circ} \mathrm{C}$ using a light-to-dark regimen of $16: 8$ h. They were collected on 14th day, during the late exponential growth phase.

Pellet and growth medium were separated by centrifugation and extracted separately. LC-MS analyses were performed in MRM mode by using the slow gradient elution mentioned above. A chromatographic peak at $11.08 \mathrm{~min}$ in the ion traces at $\mathrm{m} / \mathrm{z}$ $1340.7 \rightarrow 327.1$ and $1331.7 \rightarrow 327.1$ evidenced the presence of putative palytoxin as well as a chromatographic peak at $11.48 \mathrm{~min}$ in the ion traces at $\mathrm{m} / \mathrm{z}$ $1324.7 \rightarrow 327.1$ and $1315.7 \rightarrow 327.1$ highlighted the presence of ovatoxin-a. These data were conclusive in indicating O. ovata as the producing organism of both compounds detected in natural plankton. Quantita- tive analyses for pellet and growth medium extracts of $O$. ovata cultures afforded a putative palytoxin and an ovatoxin-a content of $0.55 \mathrm{pg} / \mathrm{cell}$ and $3.85 \mathrm{pg} /$ cell, respectively. Interestingly, the two compounds appeared to be produced approximately in the same ratio observed in natural plankton.

\section{Conclusions}

The present paper provides further insights into the toxin profile of the Mediterranean O. ovata. The reported results confirmed the presence of putative palytoxin in the Mediterranean Sea and evidenced the occurrence of the novel ovatoxin-a. This latter compound was believed to be the major toxin produced by O. ovata during the 2006 outbreak. On the basis of molecular formula (ovatoxin-a presents two oxygen atoms less than palytoxin), fragmentation pattern, and chromatographic behavior, the structure of ovatoxin-a appeared to be strictly related to that of palytoxin. However, even slight structural differences could significantly affect and differentiate the toxicology of the two compounds on the account of data reported on bio-activity of palytoxin analogues $[10,17]$. Thus, isolation of ovatoxin-a is necessary both to elucidate its chemical structure and evaluate its toxicology.

\section{Acknowledgments}

This work is a result of a research supported by BIOTOX marin 2005. LC-MS experiments on the triple quadrupole MS and on the ion trap MS were performed at Centro di Servizio Interdipartimentale di Analisi Strumentale, Università degli Studi di Napoli Federico II, and at Centro Regionale di Competenza in Diagnostica e Farmaceutica Molecolari (Regione Campania), respectively. The authors gratefully acknowledge the assistance of the staff. The authors also thank Thermo Fisher Scientific for LC-MS experiments on the linear ion trap hybrid FTMS.

\section{References}

1. Sansoni, G.; Borghini, B.; Camici, G.; Casotti, M.; Righini, P.; Rustighi, C. Fioriture algali di Ostreopsis ovata (Gonyaulacales: Dinophyceae): un problema emergente. Biologia Ambientale 2003, 17, 17-23.

2. Di Girolamo, I.; Fattorusso, E.; Funari, E.; Gramaccioni, L.; Grillo, C.; Icardi, G.; Mattei, D.; Poletti, R.; Scardala, S.; Testai, E. Linee guidaGestione del Rischio associato alle Fioriture di Ostreopsis ovata nelle coste italiane. Enacted by Consiglio Superiore di Sanità-Ministero della Salute-May 24th 2007 <http://www.ministerosalute.it/imgs/C_17_ pubblicazioni_641_allegato/pdf $>$.

3. Ciminiello, P.; Fattorusso, E. Shellfish Toxins-Chemical Studies on Northern Adriatic Mussels. Eur. J. Org. Chem. 2004, 12, 2533-2551.

4. Ciminiello, P.; Dell'Aversano, C.; Fattorusso, E.; Forino, M.; Magno, G. S.; Tartaglione, L.; Grillo, C.; Melchiorre, N. The Genoa 2005 Outbreak. Determination of Putative Palytoxin in Mediterranean. Ostreopsis ovata by a New Liquid Chromatography Tandem Mass Spectrometry Method. Anal. Chem. 2006, 78, 6153-6159.

5. Furey, A.; Garcia, J.; O'Callaghan, K.; Lehane, M.; Amandi, M. F.; James, K. J. Brevetoxins: Structures, Toxicology, and Origin. In Phycotoxins, Botana, L. M., Eds., Blackwell Publishing Professional: Ames, IA, 2007; p. $19-46$.

6. Moore, R. E.; Bartolini, G. Structure of Palytoxin. J. Am. Chem. Soc. 1981, 103, 2491-2494.

7. Cha, J. K.; Christ, W. J.; Finan, J. M.; Fujioka, H.; Kishi, Y.; Klein, L. L.; Ko, S. S.; Leder, J.; McWhorter, W. W., Jr.; Pfaff, K.-P.; Yonaga, M.; Uemura, D.; Hirata, Y. Stereochemistry of Palytoxin. Part 4. Complete Structure. J. Am. Chem. Soc. 1982, 104, 7369-71.

8. Taniyama, S.; Osamu, A.; Masamitsu, T.; Sachio, N.; Tomohiro, T.; Yahia, M.; Tamao, N. Ostreopsis sp., a Possible Origin of Palytoxin (PTX) in Parrotfish Scarus ovifrons. Toxicon. 2003, 42, 29-33. 
9. Usami, M.; Satake, M.; Ishida, S.; Inoue, A.; Kan, Y.; Yasumoto, T. Palytoxin Analogs from the Dinoflagellate Ostreopsis siamensis. J. Am. Chem. Soc. 1995, 117, 5389-5390.

10. Ukena, T.; Satake, M.; Usami, M.; Oshima, Y.; Naoki, H.; Fujita, T.; Kan, Y.; Yasumoto, T. Structure Elucidation of Ostreocin D, a Palytoxin Analog Isolated from the Dinoflagellate Ostreopsis siamensis. Biosci. Biotechnol. Biochem. 2001, 65, 2585-2588.

11. Ukena, T.; Satake, M.; Usami, M.; Oshima, Y.; Fujita, T.; Naoki, H.; Yasumoto, T. Structural Confirmation of Ostreocin-D by Application of Negative-Ion Fast-Atom Bombardment Collision-Induced Dissociation Tandem Mass Spectrometric methods. Rapid Commun. Mass Spectrom. 2002, 16, 2387-2393.

12. Lenoir, S.; Ten-Hage, L.; Turquet, J.; Quod, J. P.; Bernard, C.; Hennion, M. C. First Evidence of Palytoxin Analogues from an Ostreopsis mas- carenensis (Dinophyceae) Benthic Bloom in Southwestern Indian Ocean. J. Phycol. 2004, 40, 1042-1051.

13. Uemura, D.; Hirata, Y.; Iwashita, T.; Naoki, H. Studies on Palytoxins. Tetrahedron 1985, 41, 1007-1017.

14. Uthermöhl, H. Zur Vervolkommnung der quantitativen Phytoplankton Methodik. Mitt. Int. Ver. Theor. Angew. Limnol. 1958, 9, 1-38.

15. Penna, A.; Bertozzini, E.; Battocchi, C.; Galluzzi, L.; Giacobbe, M. G. Vila, M.; Garces, E.; Luglie, A.; Magnani, M. Monitoring of HAB Species in the Mediterranean Sea Through Molecular Methods. J. Plankton. Res. 2007, 29, 19-38.

16. Keller, M. D.; Selvin, R. C.; Claus, W.; Guillard, R. R. L. Media for the Culture of Oceanic Ultraphytoplankton. J. Phycol. 1987, 23, 633-638.

17. Uemura, D. Bioorganic Studies on Marine Natural Products-Diverse Chemical Structures and Bioactivities. Chem. Record 2006, 6, 235-248. 